\title{
Análisis de la abundancia e infección por muérdago en Sierra Fría, Aguascalientes, México
}

\author{
Analysis of the abundance and mistletoe infection \\ in Sierra Fría, Aguascalientes, México
}

\author{
Ricardo Clark-Tapia1, Brenda Torres-Bautista2, Cecilia Alfonso- \\ Corrado1, Juan I. Valdez-Hernández³, Gabriel González-Adame1, \\ Jorge Bretado-Velázquez ${ }^{1}$ y Jorge Campos-Contreras ${ }^{4}$
}

\begin{abstract}
RESUMEN
Los muérdagos, plantas hemiparasíticas, son el segundo agente de destrucción de los bosques mexicanos después del insecto descortezador, por lo que en el presente estudio se evaluó su infectación en poblaciones forestales de Sierra Fría, Aguascalientes, esto con la finalidad de: a) conocer los factores que influyen en la abundancia de muérdago, b) determinar el grado de infectación que presentan las especies hospederas, c) identificar si existe especificidad del muérdago por especie y tamaño del hospedero y/o por hábitat. En enero de 2007 se seleccionaron 20 sitios de muestreo dentro de los cuales se establecieron dos unidades de muestreo (UM) de $2500 \mathrm{~m}^{2}$. En cada UM se midió la altura y diámetro de todos los individuos (infestados y no infestados) $>1 \mathrm{~m}$ de altura y se registró la presencia de muérdago. Se registraron, además, la altitud, inclinación y orientación de la pendiente, nivel de perturbación y grado de infección. Los resultados indican que el grado de infección por muérdago muestra asociación positiva con la altitud y orientación y una asociación negativa con la perturbación. Se encontró que al aumentar en tamaño los atributos dasométricos (altura, diámetro) se incrementa el grado de infección. Phoradendron bolleanum fue la especie de muérdago más abundante, siendo su hospedero más común Juniperus deppeana. En contraste con lo mencionado en otros trabajos, las poblaciones forestales de Sierra Fría no se encuentran severamente afectadas por muérdago; sin embargo, se recomienda realizar estudios futuros que permitan comprender los factores que dan forma a la distribución e interacción hemiparásito-hospedero, dentro y entre poblaciones, con la finalidad de evaluar los daños reales o futuros, así como evaluar la selectividad hacia ciertas especies hospederas y los cambios químicos que sufren los árboles a través de la infección.
\end{abstract}

PALABRAS CLAVE:

Especificidad, hemiparásita, hospedero, perturbación, Sierra Fría.

Instituto de Estudios Ambientales, Universidad de la Sierra de Juárez. Av. Universidad s/n, Ixtlán de Juárez, Oaxaca, México. CP 68725. c.e.: rclark@juppa.unsij.edu.mx

2 Herbario, Universidad Autónoma de Aguascalientes. Av. Universidad núm. 940, Cd. Universitaria, Aguascalientes, Ags. CP 20100.

3 PF-COLPOS. CP 56230, Montecillos, Texcoco, Estado de México. Tel. (595) 9520200 ext 1484, Tel. D.F. (55) 58045900 ext 1484.

4 Facultad de Estudios Superiores Iztacala. UNAM. Unidad de Biotecnología y Prototipos. Apartado Postal 314. 54000 Tlalnepantla, Estado de México. 


\begin{abstract}
Mistletoe, a hemiparasitic plant, is the second most destructive agent for Mexican forests after the bark beetle. For this reason, in the present study, its infestation in the forest populations of Sierra Fría, Aguascalientes was examined in order to: a) understand the factors that influence the abundance of mistletoe; (b) determine the infestation level present in host species and (c) identify if there exists specific types of mistletoe that correspond to different species and sizes of the host and / or habitat. In January 2007, 20 sampling locations were selected within which two sampling units were established (UM) of $2500 \mathrm{~m}^{2}$. In every UM the height and diameter of all individuals (infected and uninfected) $>1 \mathrm{~m}$ in height were measured and the presence of mistletoe was recorded. Also recorded was the altitude, incline, slope orientation, level of disturbance and degree of infestation. The results indicate that the level of mistletoe infection shows positive association with altitude and orientation and a negative association with disturbance. It was found that as the size of dasometric attributes (height, diameter) increases so too does the degree of infection. Phoradendron bolleanum was the most abundant species of mistletoe, with Juniperus deppeana being its most common host. In contrast to that reported by other works, Sierra Fría forest populations are not found to be severely affected by mistletoe. Nevertheless, it is recommended that future studies be undertaken that allow for the understanding of the factors that shape the distribution and hemiparasitic-host interaction within and between populations, in order to assess real or future damage and to evaluate the selectivity towards certain host species and chemical changes from which trees suffer as a result of infection.
\end{abstract}

KEYWORDS:

Specificity, hemiparasitic, host plants, disturbance Sierra Fría.

\section{INTRODUCCIÓN}

Los muérdagos son plantas generalmente arbustivas o subarbustivas pertenecientes al Orden Santalales, el cual se encuentra constituido por 13 familias (sensu APG II, 2008). En este orden se encuentra la familia Santalaceae (que en la actualidad incluye a Viscaceae) representada por 151 géneros y 1985 especies, siendo Phoradendron uno de estos géneros (Simpson, 2006; Tropicos, 2011). El género Phoradendron se distribuye desde Estados Unidos de Norteamérica hasta Sudamérica (Trelease, 1916) y está representado por especies hemiparásitas fotosintéticas con haustorios que atacan al xilema de la planta que hospedan para obtener agua y sales minerales, ya que son incapaces de obtenerla directamente del suelo (Simpson, 2006).

En México, el muérdago es considerado la plaga forestal con mayor extensión en nuestro país y el segundo agente de destrucción de los bosques después del escarabajo descortezador (Geils et al., 2002; Villa, 2003), debido a que los haustorios pueden causar deformación en la madera, reduciendo la vida $y / o$ ocasionando la muerte del hospedero (Gutiérrez, 1986; López-de Buen et al., 2002). La Comisión Nacional Forestal (Conafor, 2006) sugiere que los ecosistemas forestales comprendidos en los estados de Aguascalientes, San Luis Potosí, Nuevo León y Coahuila, están siendo afectados por plantas hemiparásitas del género Phoradendron y epífitas de la familia Bromeliaceae, ocasionando daño severo e incluso la muerte del arbolado de miles de hectáreas.

En ambientes fragmentados o con cierto nivel de disturbio, es necesario considerar la selectividad de especies hospederas y la distribución espacial del parásito dentro y entre poblaciones de los hospederos. El género Phoradendron presenta especificidad a ciertas especies de hospederos, generalmente infesta a coníferas y algunas angiospermas como Quercus, Arbutus y Arctostaphylos, mostrando además una distribución frecuentemente homogénea dentro de la población (Aukema, 2003). Por lo ante- 
rior, comprender los procesos de infectación de la planta parásita ayudará, sobre todo, a elucidar los factores que dan forma a la distribución, dinámica e interacción del muérdago dentro y entre poblaciones de sus huéspedes. Esto permitiría, por un lado, evaluar los daños reales o futuros dentro de una población $y$, por el otro, comprender la selectividad hacia una especie hospedera en particular y el ensamble espacial en las comunidades, como lo sugieren Press y Phoenix (2005).

Aguascalientes es uno de los estados del país reportado por Conafor (2006) en fase de alerta por la infectación de muérdago. En dicho estado, las especies de encino y pino juegan un papel ecológico muy importante, debido a que representan $90 \%$ de los bosques templados del estado, además de que desempeñan múltiples e importantes funciones, como son la captación de agua y sostienen el mayor reservorio de flora y fauna en la entidad (Sedeso, 1993). Desafortunadamente, las poblaciones forestales de Sierra Fría han sido afectadas en el siglo pasado y en el presente por factores antropogénicos: sobreexplotación forestal, sobrepastoreo (Sedeso, 1993; Minnich et al., 1994) y naturales: plagas, enfermedades (Asociación Sierra Fría, 2002; Díaz-Núñez et al., 2006) que han reducido y transformado la cubierta forestal en esta región.

El conocimiento actual del género Phoradendron para el estado de Aguascalientes se reduce a estudios anatómicos (Gutiérrez, 1986) y taxonómicos (GarcíaRegalado, 1998). Estos trabajos brindan una idea del hospedero preferido; sin embargo, existe una total carencia de estudios ecológicos, particularmente de los que evalúan la abundancia y niveles de infectación para el estado. Gutiérrez (1986) sugiere que el disturbio presente en Sierra Fría ha incrementado los niveles de infectación por muérdago; sin embargo, su trabajo no presenta evidencia al respecto. Por lo anterior, el objetivo de este trabajo es evaluar el nivel de infectación de los muérdago presentes en los bosques de Sierra Fría, Aguascalientes, con la finalidad de: a) determinar la abundancia de individuos infestados y el grado de infección que presentan las poblaciones de Sierra Fría, b) conocer los factores que influyen en la abundancia de muérdago en Sierra Fría y c) identificar si existe especificidad del muérdago por especie y tamaño del hospedero y/o por hábitat.

\section{MATERIAL Y METODOLOGÍA}

\section{Área de estudio}

El área de estudio se encuentra en la zona natural protegida de Sierra Fría, en el margen occidental del estado de Aguascalientes. Es una cordillera situada al sur de la Sierra Madre Occidental, de la cual forma parte, con coordenadas $22^{\circ} 0^{\prime}-22^{\circ} 15^{\prime}$ latitud norte y $102^{\circ} 30^{\prime}$ $102^{\circ} 40^{\prime}$ longitud oeste (Minnich et al., 1994).

El clima del área de estudio es templado subhúmedo, con lluvias en verano, de acuerdo con la clasificación de Rzedowski (1978) corresponde a $\mathrm{C}(\mathrm{w} 1) \mathrm{b}(\mathrm{e}) \mathrm{g}$. Presenta una temperatura entre $-3^{\circ} \mathrm{C}$ y $18^{\circ} \mathrm{C}$ y con un promedio anual de precipitación de aproximadamente $600 \mathrm{~mm}$, no obstante, también encontramos en las partes bajas de Sierra Fría clima semiárido y semicálido correspondiente a $\mathrm{Bs} 1 \mathrm{kw}(\mathrm{e})$ con una temperatura promedio anual de $17^{\circ} \mathrm{C}$ (Sedeso, 1993).

La vegetación de Sierra Fría presenta una amplia distribución relacionada a la altitud. En los bosques de Sierra Fría se pueden encontrar asociaciones de encino (e.g. Quercus eduardii Trel., Q. 
potosina Trel., Q. grisea Liebm., Q. resinosa Liebm. y $Q$. sideroxyla Humb. et Bonpl.), encino-pino (e.g. Pinus leophylla Schl. et. Cham, P. teocote Schl. et. Cham, $P$. lumholtzii Rob. et Fern. y Juniperus deppeana Steud.), así como la presencia de otras especies forestales como manzanita (Arctostaphylos pungens HBK.) y madroño (Arbutus arizonica (A. Gray) Sarg. y A. glandulosa Mart et Gal.) (De la Cerda, 1999). Mientras que los dos géneros de muérdago presentes en Sierra Fría, Aguascalientes son: Phoradendron (Santalaceae) y Psittacanthus (Loranthaceae) (García-Regalado, 1998).

\section{Diseño de la investigación}

Durante el año de 2007 se seleccionaron sistemáticamente 20 sitios de muestreo con la finalidad de analizar diferentes gradientes de altitud a lo largo y ancho de Sierra Fría (Tabla 1). En cada uno de los sitios seleccionados se establecieron dos unidades de muestreo (UM) de $2500 \mathrm{~m}^{2}$ $(50 \mathrm{~m} \times 50 \mathrm{~m})$ con separación de $500 \mathrm{~m}$ entre ellas. Dentro de cada UM se identificó y estimó la abundancia tanto de las especies hospederas como de los muérdagos (Phoradendron y/o Psittacanthus) presentes en Sierra Fría. Además, se midió la altura y el diámetro de las especies hospederas con la finalidad de establecer categorías dasométricas.

En cada UM se registró la ubicación geográfica, altitud, inclinación y orientación de la pendiente, nivel de perturbación (e.g. intensidad de extracción de leña y pastoreo) y nivel de infectación. La ubicación geográfica y la altitud $(m)$ se obtuvieron utilizando un geoposicionador marca Garmin 12XL. En campo se midieron la inclinación de la pendiente con un clinómetro marca Brunton (en porcentaje) y la exposición de la misma con una brújula marca Brunton (en grados), esta última corregida con una carta topográfica (INEGI, 1996).
Para la variable perturbación se utilizó la escala cualitativa de intensidad: ausencia (cero), baja (uno), media (dos) y alta (tres), descrita por Álvarez-Moctezuma et al. (1999); mediante la observación de indicadores de actividades que deterioran el ecosistema (desmonte por tala, pastoreo, restos de heces fecales de ganado, suelo compactado, deterioro en el estrato herbáceo y presencia de especies indicadoras de disturbio). Finalmente, para evaluar el grado de infectación y evaluación de daños del muérdago, se utilizó la metodología propuesta por Hawksworth (1977) y Conafor (2005).

Esta metodología valora, en una escala semi-cuantitativa, la abundancia de muérdago, divide la copa viva del árbol en tres secciones verticales iguales y a cada una de ellas le asigna un valor de 0 a 2 en función de la mayor o menor presencia de muérdago ( 0 , ausencia; 1 , infección leve; 2, infección fuerte). Posteriormente, se obtiene la suma de los valores asignados a los tercios de la copa, resultando un valor que oscila entre 0 (ausencia de muérdago) y 6 (máxima infección). De acuerdo con los análisis de daños observados en campo, se consideró una escala modificada de Hawksworth (1977), reduciéndola a cinco categorías de grado de infección:

Grado 1. No hay presencia de muérdago. SANO

Grado 2. Sólo se observa una planta de muérdago. LEVE

Grado 3. Más de una planta de muérdago (hasta $50 \%$ de cobertura). FUERTE

Grado 4. Más de $50 \%$ de cobertura por muérdago. SEVERA.

Grado 5. Muerte del árbol por muérdago. MUERTE. 


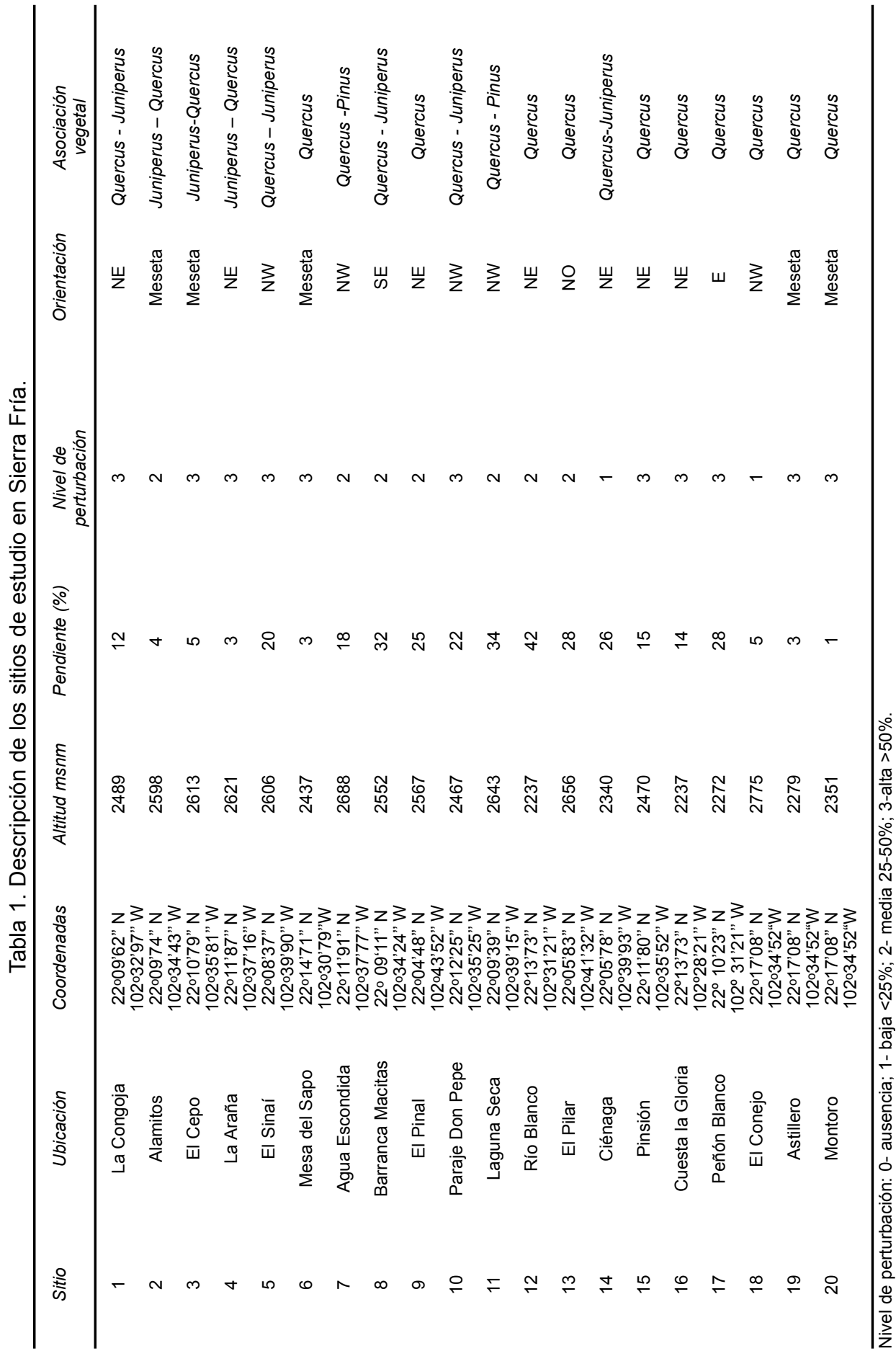


Por otro lado, las especies hospederas consideradas para cada especie de muérdago se obtuvieron con base en los registros de presencia/ausencia y fueron complementadas con el registro taxonomico de muérdagos y sus especies hospederas, realizado en Aguascalientes por García-Regalado (1998).

Por medio de análisis de varianza (ANOVA), pruebas de Chi-cuadrada y coeficientes de correlación de Spearman se obtuvo información sobre los factores que determinan la abundancia y distribución de muérdago en Sierra Fría. Estos análisis se realizaron con el programa estadístico JMP versión 4.0 (1999). Además, se realizó un análisis canónico (Ter Braak, 1988) para identificar la asociación entre las variables ambientales y las variables de infectación, utilizando el programa estadístico Statistica versión 8.0 (2007).

\section{RESULTADOS}

De los 20 sitios estudiados en Sierra Fría, 18 presentaron por lo menos un muérdago y un grado de infección leve (grado 2), mientras que dos sitios (Cuesta la Gloria y Peñon Blanco) tuvieron grado de infección 1 (sano) por ausencia de muérdago (Figura 1). En los sitios estudiados, se analizaron un total de 4004 árboles, de los cuales $313(7,81 \%)$ individuos estuvieron infectados. Por otro lado, el número de muérdago presente en las áreas de estudio fue de 1785 plantas.

Se encontró una correlación significativa entre el grado de infección y el número de muérdago presentes en una población $(r=0,513, P>0,02)$. El grado de infectación promedio de la Sierra Fría es leve (promedio 1,53 $\pm 0,74$ ) con rangos de leve (i.e. La Congoja, Alamitos, Mesa del Sapo, Agua Escondida y Paraje Don Pepe), a fuerte (El Cepo y La Araña). El grado de infección entre sitios difiere significativamente $(H=32,413, P<0,001)$, siendo las localidades centrales las que presentan una mayor abundancia de muérdago y las únicas en el estudio con grado 2 (fuerte) de infección.

Los sitios analizados presentaron un nivel de perturbación de media a alta (promedio $\pm S D=2,5 \pm 0,6$ ), altitudes mayores a los $2200 \mathrm{msnm}$, pendientes que varían de 1 hasta 42, con un promedio de $10,7 \% \pm 3 \%$ y una exposición predominante hacia el norte (NE y NW), así como una asociación de vegetación dominante de Quercus-Juniperus, Juniperus-Quercus o Quercus (Tabla 1). Los resultados del análisis canónico indican una asociación positiva $(R=0,745$, $\left.x^{2}=20,87, P=0,013\right)$ de dos variables ambientales (altitud y orientación) con tres variables de infectación (número de individuos infestados, número de muérdagos y grado de infección). El análisis canónico sugiere además una asociación negativa de la perturbación con las variables de infectación (figuras 2 y 3). La pendiente no mostró asociación con las variables de infectación $(R=0,367$, $x^{2}=2,38, P=0,49$ ).

Por otra parte, los resultados indican diferencias significativas $(H=9,55$; $\mathrm{P}<0,05)$ en el grado de infectación entre las categorías dasométricas. Las categorías de mayor diámetro presentan una abundancia más elevada de muérdago y una mayor infección (grado 2 y 3). En cambio, la categoría de $0 \mathrm{~cm}$ a $2 \mathrm{~cm}$ presenta baja abundancia de muérdago y una infección leve (grado 1) (Figura 3). De igual manera, se encontraron diferencias significativas en el grado de infección entre categorías de altura $(\mathrm{H}=17,89$, $\mathrm{P}<0,001)$. Las categorias mayores a 4 metros presentaron la mayor abundancia de muérdago y un mayor grado de infección $(2$ y 3 ) que las categorías menores a 4 metros de altura (grado 1 y 2).

Finalmente, en los sitios estudiados se presentaron cuatro especies de muér- 


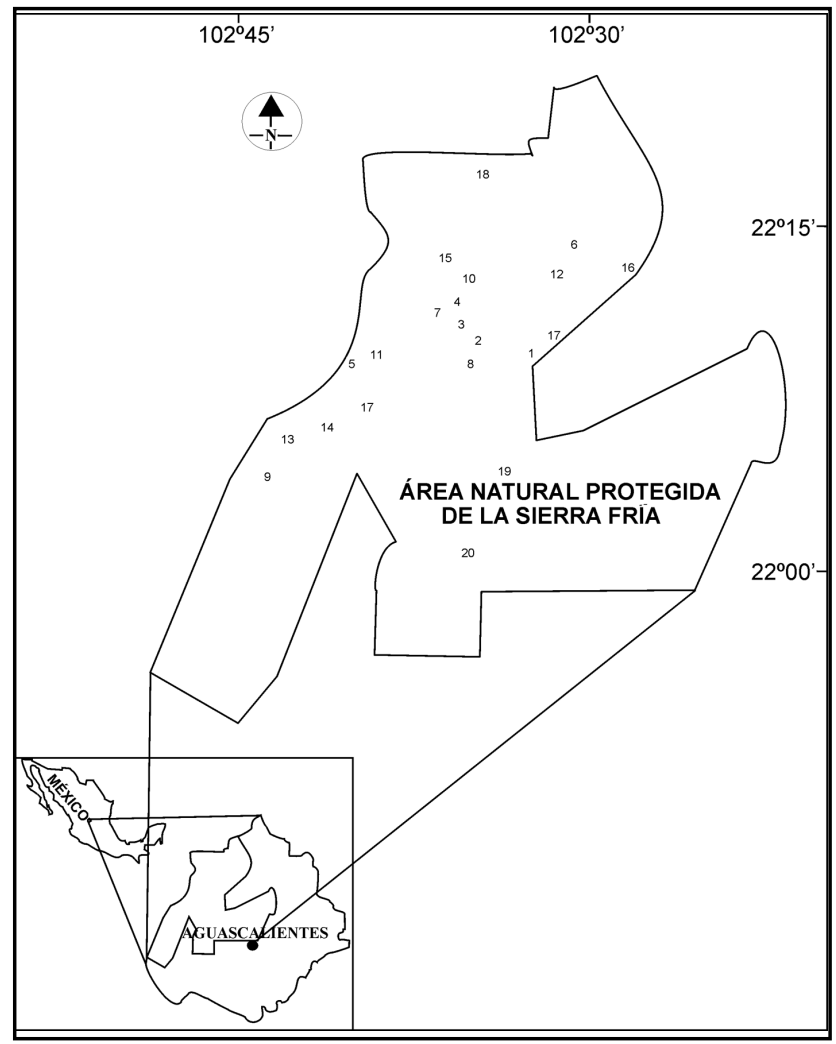

Figura 1. Sitios de estudio y localización de zonas de infectación en Sierra Fría.

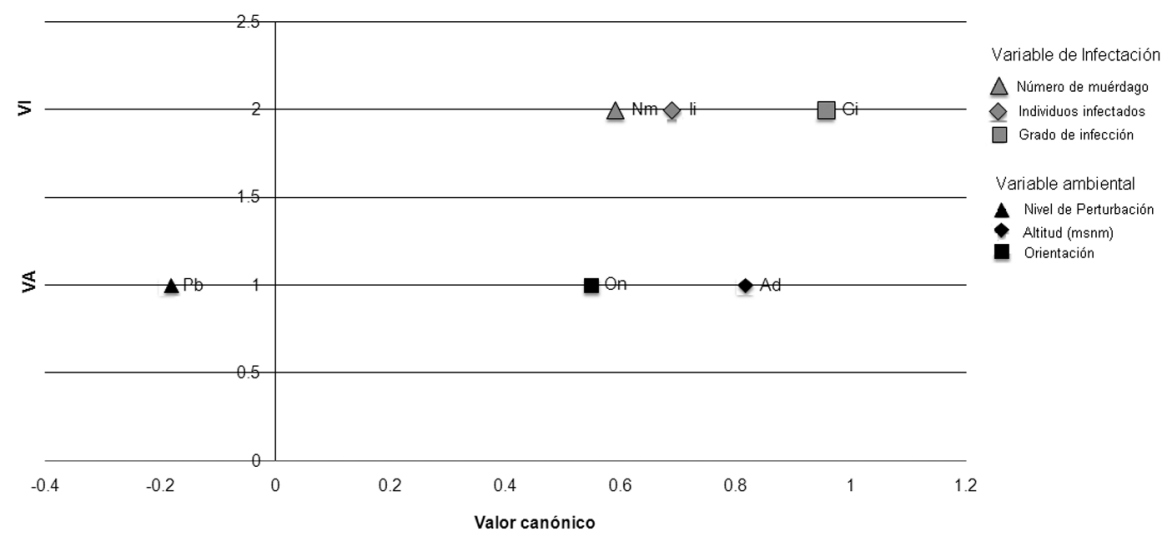

Figura 2. Análisis de correspondencia canónica (una raíz $R=0,745, x^{2}=20,87, P=0,013$ ) de las variables ambientales y variables de infectación por muérdago en Sierra Fría. 

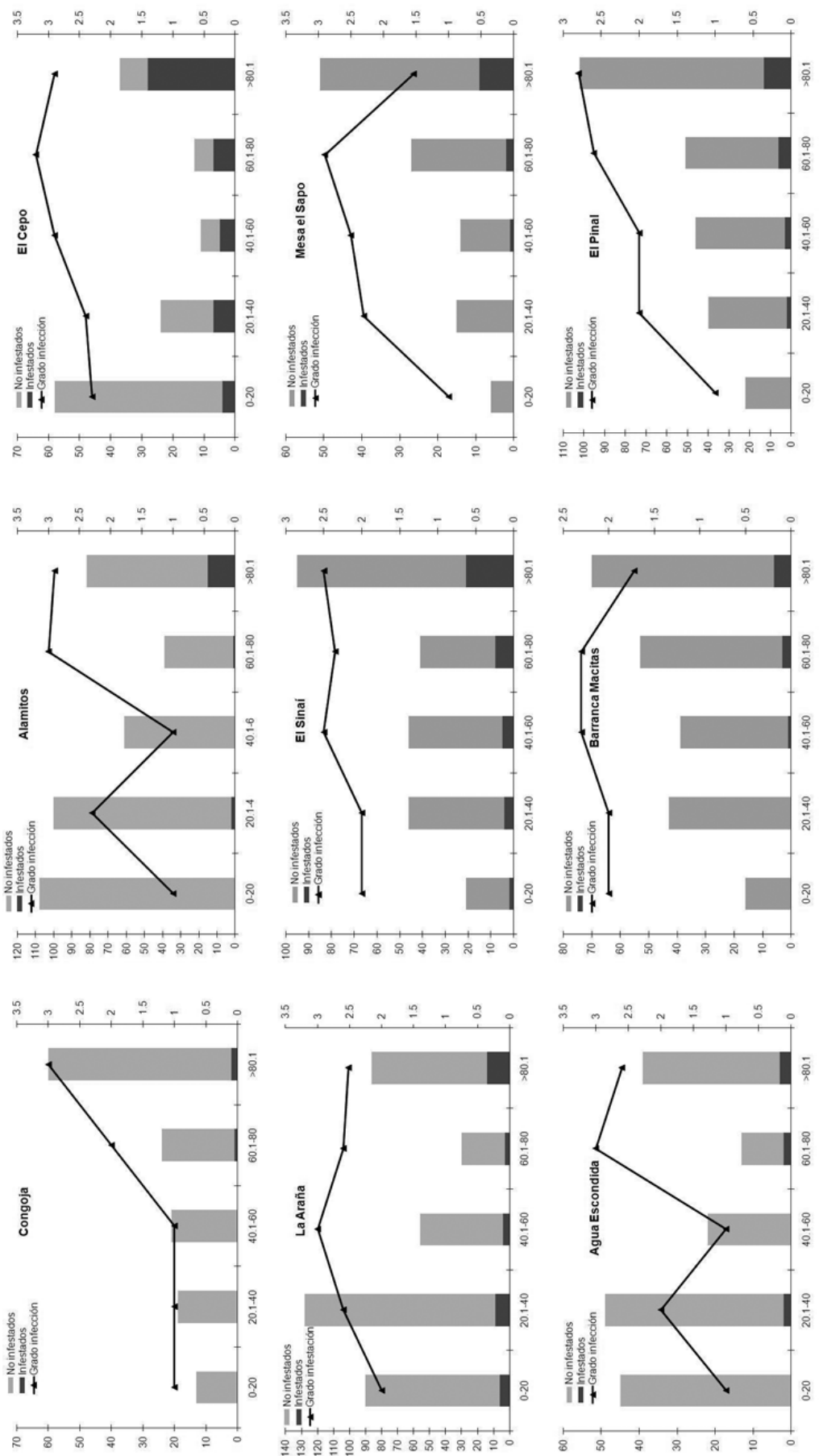

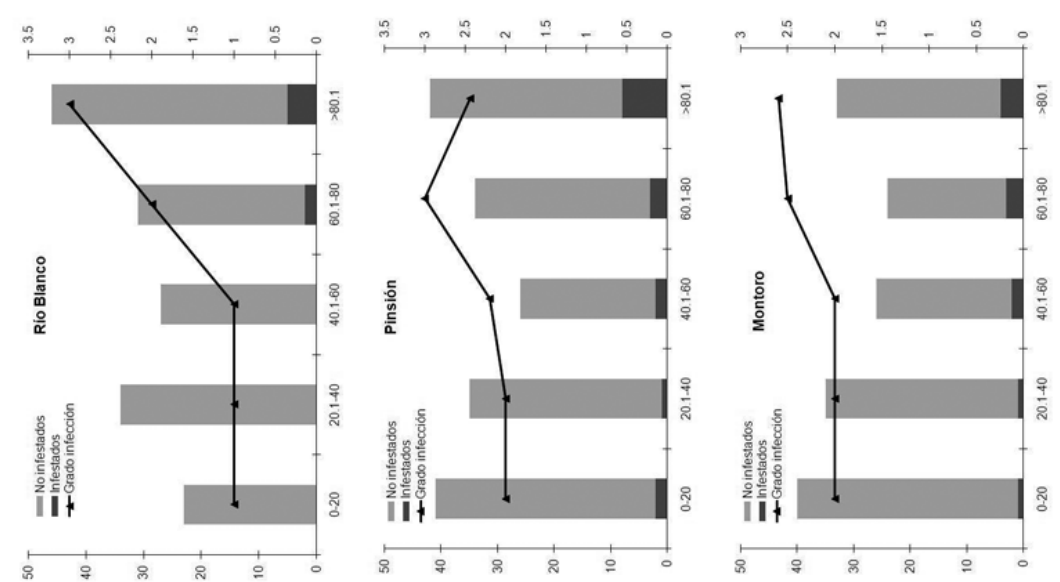

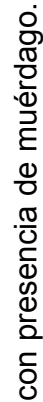
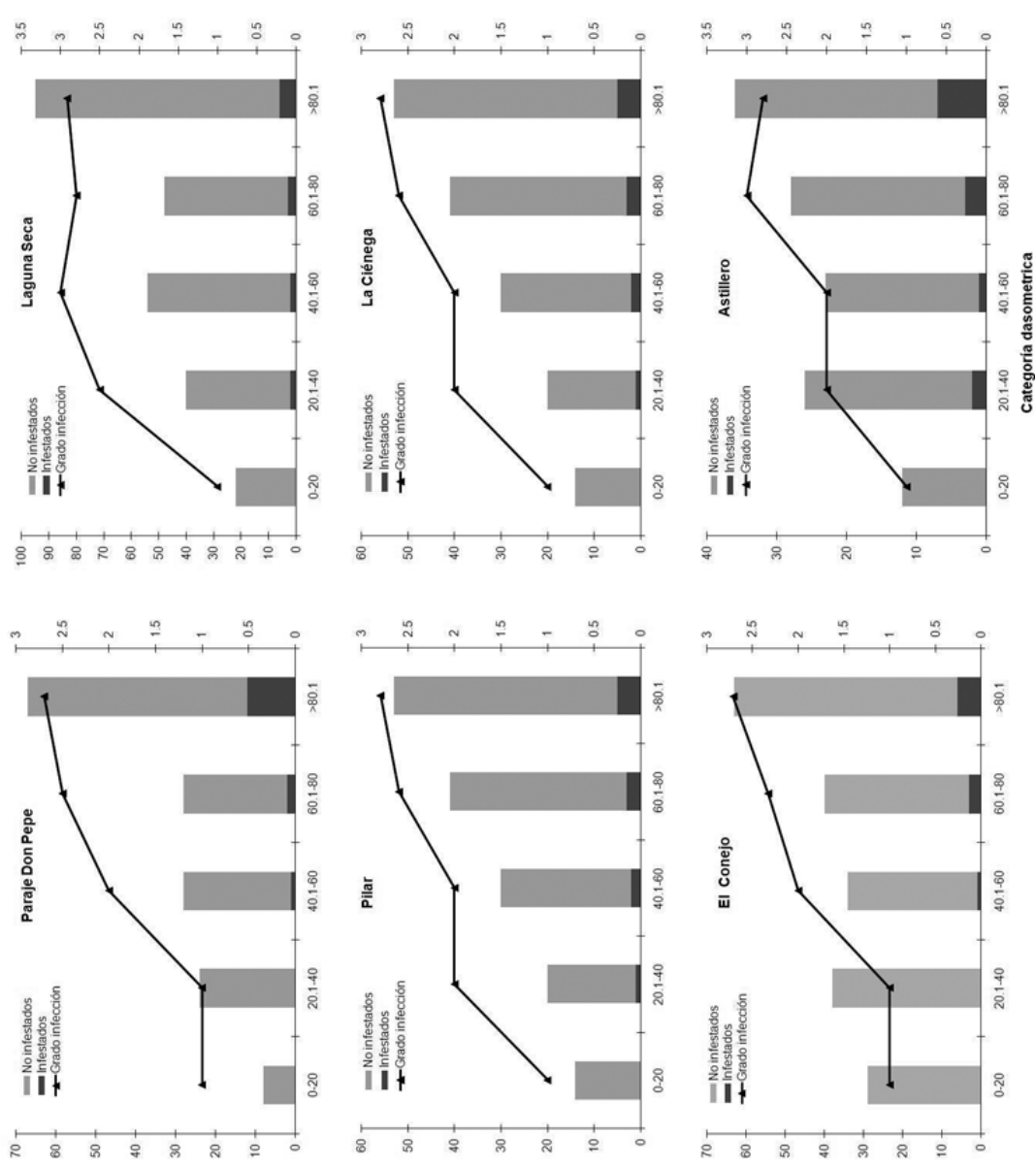

.

. 
Tabla 2. Especificidad del género Phoradendron hacia el hospedero, en Sierra Fría, Aguascalientes.

\begin{tabular}{|c|c|c|c|}
\hline Hemiparásita & Hospedero & Tipo de vegetación & Altitud \\
\hline P. bolleanum & $\begin{array}{c}\text { Juniperus deppeana, Arbutus arizonica, } \\
\text { A. glandulosa, Arctostaphylos pungens, } \\
\text { Quercus potosina. }\end{array}$ & Juniperus- Quercus & $2400-2600$ \\
\hline P. schumanni & Q. potosina, Q. eduardii y Q. sideroxyla & Quercus & $2250-2550$ \\
\hline P. villosum & Q. grisea, Q. potosina & Quercus & $2200-2600$ \\
\hline P. reichenbachinum & Q. potosina y Q. rugosa & Quercus-Pinus & $2250-2400$ \\
\hline
\end{tabular}

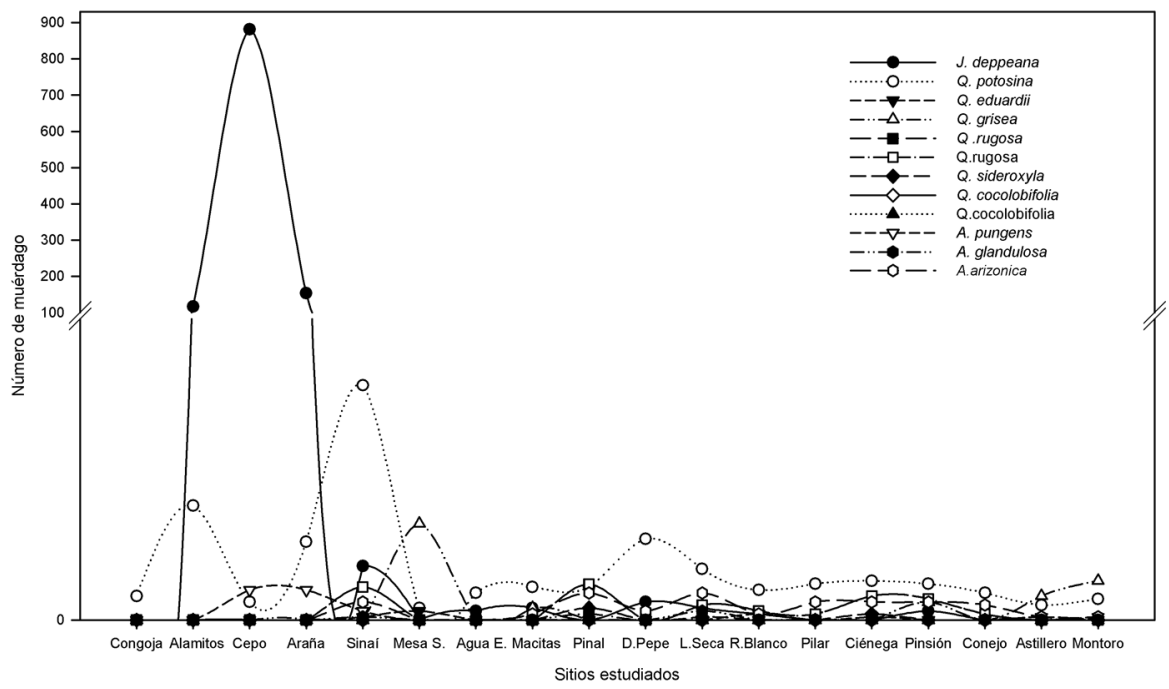

Figura 4. Número de muérdago (Phoradendron) presente en las especies hospederas de los sitios infectados.

dago que pertenencen al género Phoradendron, estas tienen una alta especificidad hacia ciertos hospederos, así como diferencias en la susceptibilidad del hospedero a ser parasitado (Tabla 2). De estas especies $J$. deppeana es la especie que presenta mayor número de individuos infestados y $Q$. syderoxyla la menos infectada $\left(\mathrm{x}^{2}=23,45, \quad \mathrm{P}>0,05\right)$; siendo $Q$. potosina la única especie susceptible a ser parasitada por todas las especies de muérdago y con registro de por lo menos un muérdago en $90 \%$ de las poblaciones estudiadas (Figura 4). 


\section{DISCUSIÓN}

Los resultados sugieren que la infectación por muérdago en Sierra Fría se encuentra asociada a variables ambientales como la altitud y la orientación de las laderas. Este resultado concuerda con el obtenido en otros estudios como los de López-Sáenz (1993) e Informe Técnico de Plagas y Patología Forestal (1999), que reportan un efecto significativo en los grados de infectación debido al ambiente. Sin embargo, contradicen a Gutiérrez (1986), quien sugiere que el disturbio en Sierra Fría ha favorecido los grados de infección por muérdago en las especies forestales.

A pesar que la perturbación no se encuentra asociada a la infectación por muérdago en Sierra Fría, esta no solo puede afectar la estructura interna del bosque, sino también la interacción parásito-hospedero (López-de Buen et al., 2002). Sierra Fría tiene una larga historia de disturbio ya que estuvo sujeta a una intensa explotación forestal y fue utilizada como área de pastoreo hasta finales del siglo XX (Minnich et al., 1994) cuando se decretó Área Natural Protegida en 1994 (Sedeso, 1993); no obstante, el disturbio ha continuado debido a la extracción ilegal de madera y al pastoreo extensivo, esto a pesar de ser Área Protegida.

En este sentido, la historia de disturbio, pasada y presente, en Sierra Fría ha propiciado una reestructuración de la cubierta y con ello un posible cambio en las preferencias del hospedero. Se conoce que la especificidad del muérdago responde a condiciones fisiológicas y químicas que propician hospederos específicos (Aukema, 2003). Se observó una alta especificidad del género Phoradendron a ciertos hospederos (i.e. Quercus y Juniperus), lo que concuerda con lo reportado por Gutiérrez (1986). Sin embargo, también se encontró que en ausencia o menor abundancia del hospe- dero específico, el muérdago puede infestar a otras especies.

Se sugiere que la infección de muérdago a otras especies puede ocurrir cuando un sitio se encuentra perturbado. En este estudio se encontró que la selección del hospedero puede variar con el tipo de vegetación y la historia de disturbio del sitio. A nivel intraespecífico, J. deppeana es la especie que presenta el grado más alto de infectación. Sin embargo, cuando $J$. deppeana presenta bajas densidades o se encuentra ausente, Phoradendron bolleanum utiliza como hospederos a especies diferentes como Arbutus arizonica, A. glandulosa, Arctosthaphylos pungens y Quercus potosina, siendo el grado de infectación en estas especies menor al encontrado en J. deppeana. De estas especies, Q. potosina es única, ya que puede ser susceptible de ser infestada por todas las especies de Phoradendron de Sierra Fría, con grados de infectación que van de leve a fuerte.

La distribución actual que presentan las especies en Sierra Fría no corresponde a su distribución pasada. Minnich et al. (1994) sugiere que J. deppeana colonizó grandes extensiones de terreno durante el siglo XX después de las extracciones de encino, particularmente en la porción central de Sierra Fría; mientras que $Q$. potosina, una de las especies más dominantes junto a $Q$. eduardii, presentó una gran revegetación. El cambio en la estructura vegetal asociada al disturbio histórico puede ser una de las razones del porqué la porción central de Sierra Fría (dominada por Juniperus y Q. potosina) presenta la mayor infectación por muérdago, esto asociado quizás a un corredor de dispersión biológico (e.g. aves) y/o a mecanismos de baja resistencia y susceptibilidad de las especies (i.e. poco grosor y resistencia de la corteza, textura suave, etc.) que permiten la penetración de los haustorios (Downey, 2004). 
Diversos estudios sugieren una asociación entre el tamaño del hospedero y el nivel de infectación (Downey, 2004; Mora, 2005). Esta asociación ha sido relacionada con la preferencia de las aves por árboles de mayor tamaño para perchar (Norton y Reid, 1997), ya que estos les brindan mayor protección que árboles de menor altura y diámetro. La selección de árboles grandes para perchar otorgan una mayor probabilidad de recibir más semillas de muérdago (Overton, 1994; Donohue, 1995), generando una distribución restringida y consecuentemente pocos árboles infestados dentro de las poblaciones, lo que concuerda con lo obtenido en este estudio. Sin embargo, es necesario realizar futuras investigaciones conducentes a evaluar corredores de dispersión para conocer las causas de las variaciones en los niveles de infectación entre poblaciones.

Asimismo, la susceptibilidad de un hospedero a ser infestado por una planta parásita está determinada por las condiciones ambientales del hábitat, por las características de la superficie del tronco, ramas y de los compuestos químicos de estos (Benzing, 1990; García-Franco, 1996). Se ha observado que cortezas lisas son menos propensas a albergar a plantas epífitas 0 a infestarse que cortezas rugosas (Botto-Mahan et al., 2000). La región central de Sierra Fría, zona de mayor infectación, está dominada por especies con tallo rugoso (J. deppeana y $Q$. potosina) por lo que se esperaría que esta hipótesis fuese correcta. Sin embargo, es necesario profundizar al respecto con nuevos estudios, ya que especies de encino con tallo rugoso como $Q$. resinosa y $Q$. rugosa presentan valores bajos de infectación, incluso similares a los de especies con tallos lisos. Futuros estudios permitirían evaluar este aspecto y determinar si las especies presentan algún mecanismo de defensa que impida el establecimiento del muérdago.

\section{CONCLUSIONES}

Las poblaciones de Sierra Fría presentan en general una leve infectación por muérdago, siendo las poblaciones centrales las que presentan los niveles más altos. La infectación por muérdago en Sierra Fría no muestra correlación con el nivel de disturbio, sin embargo, la historia de disturbio en Sierra Fría ha propiciado una reestructuración de la cubierta vegetal generado un cambio en la selección de los hospederos.

Las especies que presentan una mayor infección son Juniperus deppeana y Quercus potosina. La mayor cantidad de individuos infestados de J. deppeana se encuentran en la porción central de Sierra Fría, mientras que la infectación de individuos de $Q$. potosina es proporcional en Sierra Fría. Se encontró, además, una infectación superior en individuos de mayor diámetro y altura, y una especificidad del muérdago por ciertas especies.

En Sierra Fría, las masas forestales presentan un grado de infección de leve a fuerte (grado 2 y 3 ) y concuerdan con las observaciones realizadas por Gutiérrez (1986). De acuerdo con Conafor (2005), este grado de infección es susceptible de tratamiento de poda, por esto se recomienda realizar podas de saneamiento en las áreas más afectadas por muérdago, por ejemplo la zona central de Sierra Fría.

Se recomienda, además, realizar estudios que permitan comprender los factores que dan forma a la distribución e interacción hemiparásito-hospedero, dentro y entre poblaciones, con la finalidad de evaluar los daños reales o futuros, así como evaluar la selectividad hacia ciertas especies hospederas y los cambios químicos que sufren los árboles a través de la infección. 


\section{RECONOCIMIENTOS}

Agradecemos a los revisores de la revista Madera y Bosques por sus valiosos comentarios que enriquecieron este trabajo. Asimismo, agradecemos a Gerardo Regalado y Margarita de la Cerda Lemus, responsables del herbario de la Universidad Autónoma de Aguascalientes, por las facilidades brindadas durante el desarrollo del trabajo e identificación de las especies de muérdago y especies forestales, y a Tania Sánchez por el apoyo en campo durante el estudio. A Conafor-Conacyt, por el apoyo financiero otorgado al proyecto 14704 .

\section{REFERENCIAS}

Álvarez-Moctezuma, J.G., S. Ochoa-Gaona, B.H.J. de Jong y M.L. Soto-Pinto. 1999. Hábitat y distribución de cinco especies de Quercus (Fagaceae) en la Meseta Central de Chiapas, México. Revista de Biología Tropical 47:351-358.

APG II. 2008. Angiosperm Phylogeny Group: an update of the angiosperm phylogeny group classification for the orders and families of flowering plants: APG II. http://www.mobot.org/ мовот/research/APweb. Consulta 15 Feb 2011.

Asociación Sierra Fría (A.S.F.). 2002. La lucha contra el descortezador de pino (Dendroctonus spp.) en la Sierra Fría, Aguascalientes (Informe sometido a la embajada estadunidense en México). 18 p.

Aukema, J.E. 2003. Vectors, viscin and Viscaceae: mistletoes as parasites, mutualists, and resources. Front. Ecol. Environ. 1:212- 219.

Benzing, D.H. 1990. Vascular epiphytes. General biology and related biota.
Cambridge University Press, Nueva York.

Botto-Mahan, C., R. Medel, R. Ginocchio y G. Montenegro. 2000. Factores que afectan la distribución circular del muérdago sin hojas Tristerix aphyllus (Loranthaceae) sobre el cacto Echinopsis chilensis. Rev. chil. hist. nat. 73:525-531.

CONAFOR, 2005. Manual de Sanidad Forestal. Gerencia de Sanidad Forestal. Coordinación General de Conservación Forestal. 51 p.

CONAFOR, 2006. Fondo Sectorial para la Investigación, el Desarrollo y la Innovación Tecnológica Forestal. Demanda 1.11 Ecología y manejo de plantas parásitas y epífitas en ecosistemas forestales del semidesierto mexicano. http://www.conacyt. $\mathrm{mx} /$ fondos/FondosSectoriales/CON AFOR/Convocatoria \%20CONAFORCONACYT\%20200601/CONAF OR_Demandas_2006-01.pdf.

De la Cerda, L.M. 1999. Encinos de Aguascalientes. Universidad Autónoma de Aguascalientes, México.

Díaz-Núñez, V., G. Sánchez-Martínez y E.N. Gillete. 2006. Respuesta de Dendroctonus mexicanus (Hopkins) a dos isómeros ópticos de verbenona. Agrociencia 40:349-354.

Donohue, K. 1995. The spatial demography of mistletoe on a Yemeni acacia. International Journal Plant Science 156:816-823.

Downey, P.O. 2004. A regional examination of the mistletoe host species inventory. Cunninghamia 8:354-361.

García-Franco, J.G. 1996. Distribución de epífitas vasculares en matorrales 
costeros de Veracruz, México. Acta Botánica Mexicana 37:1-9.

García-Regalado, G. 1998. La Familia Loranthaceae (injertos) del estado de Aguascalientes, México. Polibotánica 7:1-14.

Geils, B.W., J.T. Cibrián, B. Moody. 2002. Mistletoes of North American Conifers. Gen. Tech. Rep. RMRS-GTR-98. Ogden, UT: U.S. Department of Agriculture, Forest Service, Rocky Mountain Research Station. 123 p.

Gutiérrez, R.G. 1986. Estudio del comportamiento del muérdago sobre Quercus spp. En la Sierra de San Blas de Pabellón del Estado de Aguascalientes. Tesis de Licenciatura. Universidad Autónoma de Aguascalientes.

Hawksworth, F.G. 1977. The 6 class Dwarf Mistleote Rating System. USDA Forest Service. General Technical Report RM48. Rocky Mountain Forest and Range Experiment Station Forest service U.S. Departament of Agriculture, fort Collis, Colorado. $7 \mathrm{p}$.

INEGI, 1996. Carta topográfica Escala 1:50 000. Instituto Nacional de Estadística, Geografía e Informática. Secretaría de Programación y Presupuesto. México, D.F.

Informes Técnicos de Plagas y Patología Forestal. 1999. Inventario de árboles afectados por muérdago (Viscum album) en el Monte Herbeset del término municipal de Morelia. Resultados y propuestas de gestión. Sección de estudios y planificación forestal.

López-de Buen, L., J.F. Ornelas y J.G. García-Franco. 2002. Mistletoe infection of trees located at fragmented forest edges in the cloud forest of Central Veracruz, Mexico.
Forest Ecology and Management 164:293-302.

López-Sáez, J.A. 1993. Contribución a la corología y ecología del muérdago (Viscum album L.) en el centro y norte de la Península Ibérica. Boletín de Sanidad Vegetal-Plagas 19:551-558. Madrid.

Minnich, R., J. Sosa Ramírez, E. FrancoVizcaíno, J.W Barry y M.E. Siqueiros. 1994. Reconocimiento preliminar de la vegetación y de los impactos de actividades humanas en la Sierra Fría, Aguascalientes, México. Investigación y Ciencia. Universidad Autónoma de Aguascalientes. Aguascalientes. Investigación y Ciencia 4:23-29.

Mora M. 2005. Studies of the population ecology of the mistletoe Phoradendron anceps (Viscaceae) and its host Pisonia albida (Nyctaginaceae) in the Guanica Forest Reserve, Puerto Rico. Master in Science, University of Puerto Rico.

Norton, D.A. y N. Reid. 1997. Lessons in ecosystem management from management of threatened and pest Loranthaceous mistletoes in New Zeland and Australia. Conservation Biology 11:759-769.

Overton, J.M. 1994. Dispersal and infection in mistletoe metapopulations. Journal of Ecology 82:711-723.

Press, M.C. y K.P. Phoenix. 2005. Impacts of parasitic plants on natural communities. New Phitologist 160:737-751.

Rzedowski, J. 1978. Vegetación de México. México, Limusa. México. 504 pp

Simpson, M.G. 2006. Plant Systematics. Elsevier Press. Canada. 249. 
Trelease, W. 1916. The Genus Phoradendron, a monographic revision. University or Illinois. EUA.

Ter Braak, C.J.F. 1988. Canonical correspondence analysis: a new eigenvector technique for multivariate direct gradient analysis. Ecology 67:1167-79.

Trópicos, 2011. Missouri Botanical Garden. http://www.tropicos.org. Consulta 15 Feb 2011.
SEDESO. 1993. Estudio para la declaratoria de la Sierra Fría como área natural protegida (Volumen 2). Primera edición, SEDESO. Aguascalientes.

Villa, C.J. 2003. Importante contribución a la salud de ecosistemas forestales. Forestal XXI Noviembre-Diciembre. Pp. 27-28. 